\title{
Three-Loop Corrections to the Soft Anomalous Dimension in Multileg Scattering
}

\author{
Øyvind Almelid, ${ }^{1}$ Claude Duhr, ${ }^{2,3, *}$ and Einan Gardi ${ }^{1}$ \\ ${ }^{1}$ Higgs Centre for Theoretical Physics, School of Physics and Astronomy, The University of Edinburgh, \\ Edinburgh EH9 3FD, Scotland, United Kingdom \\ ${ }^{2}$ CERN Theory Division, 1211 Geneva 23, Switzerland \\ ${ }^{3}$ Center for Cosmology, Particle Physics and Phenomenology (CP3), Université Catholique de Louvain, \\ 1348 Louvain-La-Neuve, Belgium
}

(Received 26 May 2016; published 20 October 2016)

\begin{abstract}
We present the three-loop result for the soft anomalous dimension governing long-distance singularities of multileg gauge-theory scattering amplitudes of massless partons. We compute all contributing webs involving semi-infinite Wilson lines at three loops and obtain the complete three-loop correction to the dipole formula. We find that nondipole corrections appear already for three colored partons, where the correction is a constant without kinematic dependence. Kinematic dependence appears only through conformally invariant cross ratios for four colored partons or more, and the result can be expressed in terms of single-valued harmonic polylogarithms of weight five. While the nondipole three-loop term does not vanish in two-particle collinear limits, its contribution to the splitting amplitude anomalous dimension reduces to a constant, and it depends only on the color charges of the collinear pair, thereby preserving strict collinear factorization properties. Finally, we verify that our result is consistent with expectations from the Regge limit.
\end{abstract}

DOI: 10.1103/PhysRevLett.117.172002

Infrared singularities are a salient feature of gaugetheory scattering amplitudes, and their knowledge is key to precision collider physics. Indeed, while cross sections are finite, their perturbative computation is based on amplitudes with a fixed number of on-shell partons, which are separately infrared divergent. These divergences are at the heart of factorization properties separating the physics at different energy scales. Moreover, a detailed understanding of the divergences is needed to compute cross sections at fixed order in $\alpha_{s}$, where separate divergent components with different multiplicities are combined. Finally, the cancellation of singularities may leave behind large logarithms, which can be resummed once the structure of the corresponding singularities is determined. For these reasons, there has been a continuous theoretical interest in the factorization and exponentiation properties of the singularities and their detailed structure, starting from the analysis of the form factor early on [1-10] through to many recent studies of multileg amplitudes of both massless [9,11-28] and massive partons [29-39] at the multiloop level and the formulation of the non-Abelian exponentiation theorem in the multileg case [40-44].

In this Letter, we determine the infrared (IR) singularity structure of any scattering amplitude of $n$ colored massless partons with momenta $p_{i}$ and any number of noncolored

Published by the American Physical Society under the terms of the Creative Commons Attribution 3.0 License. Further distribution of this work must maintain attribution to the author(s) and the published article's title, journal citation, and DOI. particles to three loops. Long-distance singularities (both soft and collinear) can be factorized as follows:

$$
\mathcal{M}_{n}\left(\left\{p_{i}\right\}, \alpha_{s}\right)=Z_{n}\left(\left\{p_{i}\right\}, \mu, \alpha_{s}\right) \mathcal{H}_{n}\left(\left\{p_{i}\right\}, \mu, \alpha_{s}\right),
$$

where $\mu$ is a factorization scale, $\alpha_{s} \equiv \alpha_{s}\left(\mu^{2}\right)$ is the renormalized $D$-dimensional running coupling, $\mathcal{H}_{n}$ is a finite hard scattering function, and $Z_{n}$ is an operator in color space collecting all IR singularities in the form of poles in the dimensional regularization parameter $\epsilon=(4-D) / 2$. These singularities originate in loop momenta becoming either soft or collinear to any of the scattered partons (see, e.g., Ref. [45]). Collinear singularities depend on the spin and momentum of that particle and decouple from the rest of the process. In contrast, soft singularities are independent of the spin, but they depend on the relative directions of motion and the color degrees of freedom of the scattered particles. Hence, soft singularities are sensitive to the color flow in the entire process, and their structure is a priori rather complex. Nevertheless, they are significantly simpler than finite contributions to the amplitude. They can be computed by considering correlators of products of Wilson-line operators emanating from the hard interaction, following the classical trajectory of the scattered particles and carrying the same color charge.

Specifically, $Z_{n}$ can be obtained as a solution of a renormalization-group equation as

$$
Z_{n}=P \exp \left(-\frac{1}{2} \int_{0}^{\mu^{2}} \frac{d \lambda^{2}}{\lambda^{2}} \Gamma_{n}\left(\left\{p_{i}\right\}, \lambda, \alpha_{s}\left(\lambda^{2}\right)\right)\right),
$$


where $\Gamma_{n}$ is the so-called soft anomalous dimension matrix for multileg scattering and $P$ stands for path ordering of the matrices according to the order of scales $\lambda . \Gamma_{n}$ itself is finite, and IR singularities are generated in Eq. (2) through the dependence of $\Gamma_{n}$ on the $D$-dimensional coupling, which is integrated over the scale down to zero momentum. The functional form of $\Gamma_{n}$ is highly constrained: Owing to factorization and the rescaling symmetry of the Wilson line velocities [18-20], through three loops it must take the form

$$
\Gamma_{n}\left(\left\{p_{i}\right\}, \lambda\right)=\Gamma_{n}^{\operatorname{dip}}\left(\left\{p_{i}\right\}, \lambda\right)+\Delta_{n}\left(\left\{\rho_{i j k l}\right\}\right),
$$

with

$$
\begin{aligned}
\Gamma_{n}^{\mathrm{dip}}\left(\left\{p_{i}\right\}, \lambda\right)= & -\frac{1}{2} \hat{\gamma}_{K}\left(\alpha_{s}\right) \sum_{i<j} \log \left(\frac{-s_{i j}}{\lambda^{2}}\right) \mathbf{T}_{i} \cdot \mathbf{T}_{j} \\
& +\sum_{i=1}^{n} \gamma_{J_{i}}\left(\alpha_{s}\right),
\end{aligned}
$$

where $-s_{i j}=2\left|p_{i} \cdot p_{j}\right| e^{-\mathrm{i} \pi \lambda_{i j}}$, with $\lambda_{i j}=1$ if partons $i$ and $j$ both belong to either the initial or the final state and $\lambda_{i j}=0$ otherwise; $\mathbf{T}_{i}$ are color generators in the representation of parton $i$, acting on the color indices of the amplitude as described in Ref. [11]; $\hat{\gamma}_{K}\left(\alpha_{s}\right)$ is the universal cusp anomalous dimension [7,46,47], with the quadratic Casimir of the appropriate representation scaled out (Casimir scaling of the cusp anomalous dimension holds through three loops [46]; it may be broken by quartic Casimirs starting at four loops); $\gamma_{J_{i}}$ are the anomalous dimensions of the fields associated with external particles, which govern hard collinear singularities, currently known to three loops $[28,48]$. Equation (4) is known as the dipole formula and captures the entirety of the soft anomalous dimension up to two loops.

According to the non-Abelian exponentiation theorem [44], the color factors in $\Delta_{n}$ must all correspond to connected graphs as shown in Fig. 1. Tripole corrections correlating three partons, with color factors of the form if ${ }^{a b c} \mathbf{T}_{i}^{a} \mathbf{T}_{j}^{b} \mathbf{T}_{k}^{c}$, which could appear starting from two loops, are not present in $\Delta_{n}$ at any order because the corresponding kinematic dependence on the three momenta is bound to violate the rescaling symmetry constraints [18-20].
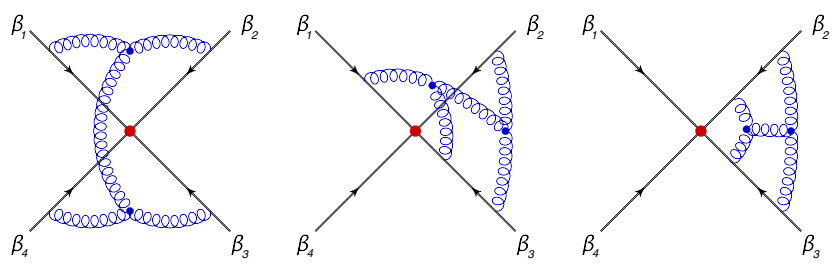

FIG. 1. Representative three-loop connected webs contributing to the soft anomalous dimension.
While a constant correction proportional to if ${ }^{a b c} \mathbf{T}_{i}^{a} \mathbf{T}_{j}^{b} \mathbf{T}_{k}^{c}$ is excluded by Bose symmetry, kinematic-independent corrections involving three lines of the form $f^{a b e} f^{c d e}\left\{\mathbf{T}_{i}^{a}, \mathbf{T}_{i}^{d}\right\} \mathbf{T}_{j}^{b} \mathbf{T}_{k}^{c}$ (last two diagrams in Fig. 1) are admissible and do indeed appear. The first admissible corrections involving kinematic dependence in Eq. (3) are then quadrupoles, because four momenta can form conformally invariant cross ratios (CICRs),

$$
\rho_{i j k l} \equiv \frac{\left(-s_{i j}\right)\left(-s_{k l}\right)}{\left(-s_{i k}\right)\left(-s_{j l}\right)}
$$

which are invariant under a rescaling of any of the momenta. Since diagrams with four color generators first appear at three loops, $\Delta_{n}$ in Eq. (3) starts at this order:

$$
\Delta_{n}\left(\left\{\rho_{i j k l}\right\}\right)=\sum_{\ell=3}^{\infty}\left(\frac{\alpha_{s}}{4 \pi}\right)^{\ell} \Delta_{n}^{(\ell)}\left(\left\{\rho_{i j k l}\right\}\right) .
$$

Three-loop graphs can connect at most four lines, so the general form of the three-loop correction is completely determined by the four-parton case and can be written as

$$
\begin{aligned}
\Delta_{n}^{(3)}\left(\left\{\rho_{i j k l}\right\}\right)= & 16 f_{a b e} f_{c d e} \\
& \times\left\{\sum _ { 1 \leq i < j < k < l \leq n } \left[\mathbf{T}_{i}^{a} \mathbf{T}_{j}^{b} \mathbf{T}_{k}^{c} \mathbf{T}_{l}^{d} \mathcal{F}\left(\rho_{i k j l}, \rho_{i l j k}\right)\right.\right. \\
& +\mathbf{T}_{i}^{a} \mathbf{T}_{k}^{b} \mathbf{T}_{j}^{c} \mathbf{T}_{l}^{d} \mathcal{F}\left(\rho_{i j k l}, \rho_{i l k j}\right) \\
& \left.+\mathbf{T}_{i}^{a} \mathbf{T}_{l}^{b} \mathbf{T}_{j}^{c} \mathbf{T}_{k}^{d} \mathcal{F}\left(\rho_{i j l k}, \rho_{i k l j}\right)\right] \\
& \left.-C \sum_{i=1}^{n} \sum_{\substack{1 \leq j<k \leq n \\
j, k \neq i}}\left\{\mathbf{T}_{i}^{a}, \mathbf{T}_{i}^{d}\right\} \mathbf{T}_{j}^{b} \mathbf{T}_{k}^{c}\right\}
\end{aligned}
$$

where $C$ is a constant and $\mathcal{F}$ is a function of two CICRs. Both $C$ and $\mathcal{F}$ are independent of the color degrees of freedom. Moreover, Eq. (7) is the most general three-loop ansatz consistent with Bose and rescaling symmetry, so $C$ and $\mathcal{F}$ are independent of the number of legs $n$. Note that the terms in this sum are not all independent because of the antisymmetry of the structure constants and the Jacobi identity. A special property at three loops is that $\Delta_{n}^{(3)}$ is independent of the matter content of the theory and is completely determined by soft gluon interactions. In particular, this implies that $\Delta_{n}^{(3)}$ is the same in QCD and in $\mathcal{N}=4$ super Yang-Mills, and it is therefore expected to be a pure polylogarithmic function of weight five. Its functional form has been constrained by considering collinear limits and the Regge limit [18-26], but it has so far remained unclear whether three-loop corrections to the dipole formula are at all present. In this Letter, we compute $\Delta_{n}^{(3)}$ and, hence, determine soft singularities of any massless multileg amplitude at three loops. Since $C$ and 
$\mathcal{F}$ can be extracted from $\Delta_{4}^{(3)}$, we restrict our computation to the case $n=4$ without imposing momentum conservation among colored particles. Before presenting the final result, we give a brief summary of the computation. A complete account of the computation will be presented in a forthcoming publication [49].

We set up the calculation of the soft anomalous dimension through the renormalization of a product of semiinfinite Wilson lines with four-velocities $\beta_{k}$, with $\beta_{k}^{2} \neq 0$. By considering nonlightlike lines, we avoid collinear singularities and obtain kinematic dependence via cusp angles $\gamma_{i j} \equiv 2 \beta_{i} \cdot \beta_{j} / \sqrt{\beta_{i}^{2} \beta_{j}^{2}}$. We eventually extract $\Delta_{n}^{(3)}$ for massless scattering by considering the asymptotic lightlike limit $\beta_{k}^{2} \rightarrow 0$, where the kinematic dependence reduces to CICRs as in Eq. (5).

In organizing the calculation, we use the non-Abelian exponentiation theorem and we compute only webs. A web can be either an individual connected diagram, as in Fig. 1, or a set of nonconnected diagrams which are related by permuting the order of gluon attachments to the Wilson lines [40-44]; representative diagrams from such webs are shown in Fig. 2. In either of these cases, the contribution to $\Delta_{4}^{(3)}$ is associated with fully connected color factors, the classification of which was done in Ref. [44]. The sum of all two-line diagrams may be written as

$$
G_{2}(1,2)=\text { dipole }-f^{a b e} f^{c d e}\left\{\mathbf{T}_{1}^{a}, \mathbf{T}_{1}^{d}\right\}\left\{\mathbf{T}_{2}^{b}, \mathbf{T}_{2}^{c}\right\} H_{2}(1,2),
$$

where "dipole" stands for a term with a color factor proportional to $\mathbf{T}_{1} \cdot \mathbf{T}_{2}$, which contributes to $\Gamma_{n}^{\text {dip }}$. The component involving four generators via anticommutators is relevant for the calculation of $\Delta_{n}$; its kinematic dependence is contained in $H_{2}(1,2)=H_{2}(2,1)$. Similarly, the sum of all three-line diagrams takes the form

$$
G_{3}(1,2,3)=f^{a b e} f^{c d e} \sum_{\substack{(i, j, k) \in(1,2,3) \\ j<k}}\left\{\mathbf{T}_{i}^{a}, \mathbf{T}_{i}^{d}\right\} \mathbf{T}_{j}^{b} \mathbf{T}_{k}^{c} H_{3}(i, j, k),
$$

with $H_{3}(i, j, k)=H_{3}(i, k, j)$. We omitted here the tripole term, proportional to $f^{a b c} \mathbf{T}_{1}^{a} \mathbf{T}_{2}^{b} \mathbf{T}_{3}^{c}$, which vanishes for lightlike kinematics where $\gamma_{i j} \rightarrow-\infty$. Furthermore, in this limit $H_{2}$ and $H_{3}$ are necessarily polynomials in $\log \left(-\gamma_{i j}\right)$. Finally, three-loop webs connecting four lines can be cast into the form
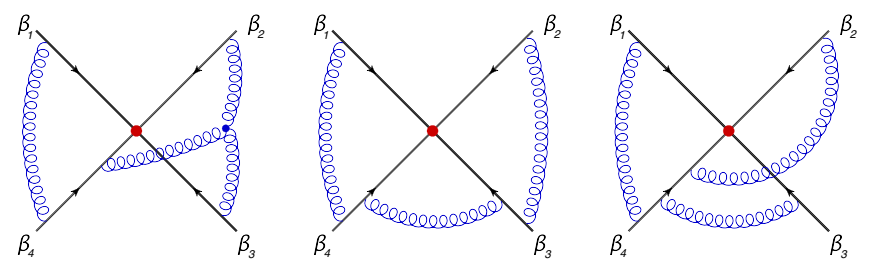

FIG. 2. Representative nonconnected three-loop diagrams of webs which contribute to $\Delta_{4}^{(3)}$.

$$
\begin{aligned}
G_{4}(1,2,3,4)= & \mathbf{T}_{1}^{a} \mathbf{T}_{2}^{b} \mathbf{T}_{3}^{c} \mathbf{T}_{4}^{d}\left[f^{a b e} f^{c d e} H_{4}(1,2,3,4)\right. \\
& +f^{a c e} f^{b d e} H_{4}(1,3,2,4) \\
& \left.+f^{a d e} f^{b c e} H_{4}(1,4,2,3)\right]
\end{aligned}
$$

where the kinematic function $H_{4}$ satisfies $H_{4}(1,2,3,4)=$ $-H_{4}(2,1,3,4)=H_{4}(3,4,1,2)$; this function depends on logarithms of cusp angles as well as on nontrivial functions of CICRs.

Another important element in organizing the calculation is color conservation. The anomalous dimension $\Gamma_{n}$ is an operator in color space that acts on the hard amplitude, which is a color singlet and must therefore satisfy [12]

$$
\left(\sum_{i=1}^{n} \mathbf{T}_{i}^{a}\right) \mathcal{H}_{n}=0
$$

Hence, when computing $\Delta_{4}^{(3)}$ one may systematically eliminate $\mathbf{T}_{4}$ in favor of $\mathbf{T}_{i}, 1 \leq i \leq 3$, thereby reducing all four-line color factors such as $f^{a b e} f^{c d e} \mathbf{T}_{i}^{a} \mathbf{T}_{j}^{b} \mathbf{T}_{k}^{c} \mathbf{T}_{l}^{d}$ to three-line ones, $f^{a b e} f^{c d e}\left\{\mathbf{T}_{i}^{a}, \mathbf{T}_{i}^{d}\right\} \mathbf{T}_{j}^{b} \mathbf{T}_{k}^{c}$. Consequently, color conservation relates sets of diagrams that connect different numbers of Wilson lines. Summing over all subsets of two and three lines out of four and using color conservation, we find that the function $\mathcal{F}$ and the constant $C$ can be expressed in terms of the kinematic functions $H_{n}$ as follows:

$$
\begin{aligned}
& \mathcal{F}\left(\rho_{i j k l}, \rho_{i l k j}\right)= H_{4}(i, j, k, l)-\frac{2}{3}\left[\bar{H}_{3}(i, j, k)-\bar{H}_{3}(i, j, l)\right. \\
&-\bar{H}_{3}(j, i, k)+\bar{H}_{3}(j, i, l)+\bar{H}_{3}(k, i, l) \\
&\left.-\bar{H}_{3}(k, j, l)-\bar{H}_{3}(l, i, k)+\bar{H}_{3}(l, j, k)\right], \\
& C=\frac{1}{3}\left[\bar{H}_{3}(i, j, k)+\bar{H}_{3}(j, k, i)+\bar{H}_{3}(k, j, i)\right],
\end{aligned}
$$

where $\bar{H}_{3}(i, j, k)=H_{3}(i, j, k)+H_{2}(i, j)+H_{2}(i, k)$. The above equations put strong constraints on the kinematic functions $H_{n}$ : The function $\mathcal{F}$ depends on CICRs, while $H_{n}$ on the right-hand side of Eq. (10) depend on logarithms of cusp angles; these must therefore conspire to combine into logarithms of CICRs. In addition, $C$ is a constant, so the kinematic dependence of the functions $H_{n}$ must cancel in the sum in Eq. (11). Our computation satisfies all these constraints, which provides a strong check of the result.

The calculation of the individual graphs is rather lengthy, and we will describe only the main steps, deferring a detailed exposition to a dedicated publication [49]. We set up the calculation in configuration space, with four nonlightlike Wilson lines with four-velocities $\beta_{k}$. The position of the three- and four-gluon vertices off the Wilson lines are integrated over in $D=4-2 \epsilon$ dimensions. Following 
Refs. [38,43], we introduce an infrared regulator which suppresses exponentially contributions far along the Wilson lines. This is necessary to capture the ultraviolet singularity associated with the vertex where the Wilson lines meet. Upon performing the integral over the overall scale, we extract an overall $1 / \epsilon$ ultraviolet pole, and the contribution to the soft anomalous dimension is the coefficient of that pole, which is finite for each of the diagrams in Fig. 1 (they have no subdivergences) and can be evaluated in $D=4$ dimensions.

Next, we observe that the integrals over the positions of the three- and four-gluon vertices give rise to one- and twoloop off-shell four-point functions, for which we derive multifold Mellin-Barnes (MB) representations. After integration over the position of the gluon emission vertex along the Wilson lines, we obtain a MB representation of each of the connected graphs for the general nonlightlike case, depending on the velocities through the cusp angles $\gamma_{i j}$. To proceed, we use standard techniques [50] to perform a simultaneous asymptotic expansion for $\gamma_{i j} \rightarrow-\infty$ corresponding to the lightlike limit, where we neglect any term suppressed by powers of $1 / \gamma_{i j}$. We then obtain a sum of lower-dimensional MB integrals. These are converted into parametric integrals using the methods of Ref. [51], which can be performed using modern integration techniques [52]. The sum over all connected graphs is expressible as a linear combination of products of logarithms of cusp angles $\gamma_{i j}$ and single-valued harmonic polylogarithms $[53,54]$ with arguments $z_{i j k l}$ and $\bar{z}_{i j k l}$, related to the CICRs (5) by

$$
z_{i j k l} \bar{z}_{i j k l}=\rho_{i j k l} \quad \text { and } \quad\left(1-z_{i j k l}\right)\left(1-\bar{z}_{i j k l}\right)=\rho_{i l k j} .
$$

We observe that individual graphs are not pure functions, but they involve pure functions of weight five multiplied by rational functions in $z_{i j k l}$ and $\bar{z}_{i j k l}$. These rational functions cancel in the sum over all connected graphs, leaving behind a pure function of weight five, in agreement with the expectation that scattering amplitudes in $\mathcal{N}=4$ super Yang-Mills have a uniform maximal weight. Moreover, mixed weight terms do appear in two-line and three-line webs but cancel out in the sum.

Adding up all the contributions, we find the following results for the function $\mathcal{F}$ and the constant $C$ :

$$
\begin{aligned}
\mathcal{F}\left(\rho_{i j k l}, \rho_{i l k j}\right) & =F\left(1-z_{i j k l}\right)-F\left(z_{i j k l}\right), \\
C & =\zeta_{5}+2 \zeta_{2} \zeta_{3},
\end{aligned}
$$

with

$$
F(z)=\mathcal{L}_{10101}(z)+2 \zeta_{2}\left[\mathcal{L}_{001}(z)+\mathcal{L}_{100}(z)\right]
$$

where $\mathcal{L}_{w}(z)$ are Brown's single-valued harmonic polylogarithms (SVHPLs) [53] (see also Ref. [55]). Note that we kept implicit the dependence of these functions on $\bar{z}$.
SVHPLs can be expressed in terms of ordinary harmonic polylogarithms (HPLs) [54] in $z$ and $\bar{z}$. The result for $F$ in terms of HPLs is attached in computer-readable format to this Letter [56].

Let us now briefly discuss the main features of the final result. First, we note that, while $F(z)$ is defined everywhere in the physical parameter space, it is single-valued only in the part of the Euclidean region (the region where all invariants are negative) where $z$ and $\bar{z}$ are complex conjugate to each other. Single-valuedness ensures that $\mathcal{F}$, and hence also $\Delta_{n}^{(3)}$, have the correct branch cut structure of a physical scattering amplitude [55,57]: It is possible to analytically continue the function to the entire Euclidean region while the function remains real throughout [58]. Next, note that if one considers $F(z)$ as a function of two independent variables $z$ and $\bar{z}$ (not a complex conjugate pair) this function has branch points for $z$ and $\bar{z}$ at 0,1 , and $\infty$. Crossing symmetry, i.e., crossing some momenta from the final to the initial state, is realized by taking monodromies around these points. This determines the analytic continuation of the function $\mathcal{F}$ from the Euclidean to the physical scattering region.

Next, let us discuss the symmetries of the final answer for the three-loop corrections to the soft anomalous dimension. In the four-line case, Bose symmetry is realized on the function $\mathcal{F}$ of CICRs by the action of the group $S_{3}$ which keeps the momentum $p_{1}$ fixed and permutes the remaining three momenta. This group naturally acts on the space of SVHPLs by a change of arguments generated by the transformations $(z, \bar{z}) \mapsto(1-\bar{z}, 1-z)$ and $(z, \bar{z}) \mapsto$ $(1 / \bar{z}, 1 / z)$, with $z \equiv z_{1234}$. Geometrically, this symmetry simply acts by exchanging the three singularities at $z \in\{0,1, \infty\}$. Moreover, it is known that the space of all HPLs, and hence also SVHPLs, is closed under the action of this $S_{3}$, giving rise to functional equations among HPLs, i.e., relations among HPLs with different arguments. Consequently, it is possible to express all the terms in Eq. (14) in terms of SVHPLs with argument $z$.

An additional symmetry group $\mathbb{Z}_{2}$ arises from the definition of $(z, \bar{z})$ in Eq. (12), which is invariant under the exchange $z \leftrightarrow \bar{z}$. Hence, $F(z)$ must be invariant under this transformation; i.e., it is even: $F(\bar{z})=F(z)$. This symmetry is realized on the space of SVHPLs by the operation of reversal of words; namely, if $w$ is a word made out of 0's and 1's and $\tilde{w}$ the reversed word, then we have $\mathcal{L}_{w}(\bar{z})=\mathcal{L}_{\tilde{w}}(z)+\cdots$, where the dots indicate terms proportional to multiple zeta values. Even functions then correspond to "palindromic" words (possibly up to multiple zeta values), and indeed Eq. (14) is palindromic.

Let us now comment on the momentum-conserving limit of $\Delta_{4}^{(3)}$, which is of particular interest because it corresponds to two-to-two massless scattering. In this limit we have $\bar{z}=z=s_{12} / s_{13}=-s /(s+t)$. It follows that for twoto-two massless scattering $F(z)$ can be expressed entirely in terms of HPLs with indices 0 and -1 depending on $s / t$, in 
agreement with known results for on-shell three-loop fourpoint integrals $[39,59,60]$. Furthermore, specializing to the Regge limit and expanding Eq. (14) at large $s /(-t)$ [61], we find no $\alpha_{s}^{3} \ln ^{p}[s /(-t)]$ for any $p>0: \operatorname{Re}\left[\Delta_{4}^{(3)}\right]$ simply tends to a constant in this limit. This is entirely consistent with the behavior of a two-to-two scattering amplitude in the Regge limit [23,24,62]; indeed, the dipole formula alone is consistent with predictions from the Regge limit for the real part of the amplitude through next-to-next-toleading logarithms at three loops [62].

Finally, let us comment on the behavior of $\Delta_{n}^{(3)}$ in the limit where two final-state partons become collinear. A well-known property of an $n$-parton scattering amplitude is that the limit where any two colored partons become collinear can be related to an $(n-1)$-parton amplitude:

$\mathcal{M}_{n}\left(p_{1}, p_{2},\left\{p_{j}\right\} \stackrel{1 \| 2}{\longrightarrow} \mathbf{S p}\left(p_{1}, p_{2}\right) \mathcal{M}_{n-1}\left(P,\left\{p_{j}\right\}\right)\right.$,

where $P=p_{1}+p_{2}$ and $p_{j}$ are the momenta of the $(n-2)$ noncollinear partons. The splitting amplitude $\mathbf{S p}\left(p_{1}, p_{2}\right)$ is an operator in color space which captures the singular terms for $P^{2} \rightarrow 0$. All elements in Eq. (15) have infrared singularities, and these must clearly be related. Furthermore, Sp is expected to depend only on the quantum numbers of the collinear pair [63] to all orders in perturbation theory and, hence, also its soft anomalous dimension

$$
\Gamma_{\mathbf{S p}}=\left.\left(\Gamma_{n}-\Gamma_{n-1}\right)\right|_{1 \| 2}=\Gamma_{\mathbf{S p}}^{\mathrm{dip}}+\Delta_{\mathbf{S p}}
$$

must be independent of the momenta and color degrees of freedom of the rest of the process. This property is automatically satisfied for the dipole formula, but it is highly nontrivial for it to persist when quadrupole corrections are present, as these might introduce correlations between the collinear pair and the rest of the process. In Refs. [19,22], this property was used to constrain $\Delta_{n}$, but this was done under the assumption that $C$ in Eq. (7) vanishes. Given our result for $\Delta_{n}^{(3)}$, we may now compute the nondipole correction to the splitting amplitude at three loops:

$$
\begin{aligned}
\Delta_{\mathbf{S p}}^{(3)}= & \left.\left(\Delta_{n}^{(3)}-\Delta_{n-1}^{(3)}\right)\right|_{1 \| 2}=-24\left(\zeta_{5}+2 \zeta_{2} \zeta_{3}\right) \\
& \times\left[f^{a b e} f^{c d e}\left\{\mathrm{~T}_{1}^{a}, \mathrm{~T}_{1}^{c}\right\}\left\{\mathrm{T}_{2}^{b}, \mathrm{~T}_{2}^{d}\right\}+\frac{1}{2} C_{A}^{2} \mathrm{~T}_{1} \cdot \mathrm{T}_{2}\right] .
\end{aligned}
$$

We note that $\Delta_{\mathbf{S p}}^{(3)}$ depends only on the color degrees of freedom of the collinear pair and is entirely independent of the kinematics and, hence, fully consistent with general expectations $[63,64]$. We emphasize that $\Delta_{\mathbf{S p}}^{(3)}$ is independent of the value of $n$ that was used to compute it, which is remarkable. Indeed, the fact that the difference in Eq. (17) is independent of $n$ requires intricate relations between different sets of diagrams and thus provides a highly nontrivial check of the calculation.

In summary, we have computed all graphs contributing to the soft anomalous dimension in multiparton scattering and determined the first multiparticle correlations going beyond the dipole formula. We find that these appear already for three colored partons in the form of a nontrivial color structure multiplied by a constant of weight five. Starting from four colored partons, multiparticle correlations involve kinematic dependence via conformally invariant cross ratios with a remarkably simple functional form in terms of single-valued harmonic polylogarithms of uniform weight five.

In conclusion, we have determined the complete threeloop soft anomalous dimension, which is the last ingredient needed to predict long-distance singularities to this order. As such, our result provides a strong constraint on multileg amplitudes at this order, and it will therefore serve as a check for any such future computation. It is also an essential ingredient in the development of future subtraction schemes for fixed-order computations and provides the basis for the resummation of large logarithms at high accuracy. Beyond these immediate applications, our result constitutes a first step into the unexplored analytic structure of multileg scattering amplitudes at three loops. In particular, it sheds light on the behavior of amplitudes in both the Regge limit and collinear limits.

The authors are grateful to S. Caron-Huot for clarifying discussions on the role of color conservation. We thank L. Dixon, G. Falcioni, M. Harley, L. Magnea, J. Pennington, J. Smillie, M. Schwartz, and C. White for useful discussions and collaboration on related topics. C. D. acknowledges the hospitality of the Higgs Centre of the University of Edinburgh at various stages of this work. This research is supported by the STFC Consolidated Grants "Particle Physics at the Tait Institute" and "Particle Physics at the Higgs Centre" (E. G.) and by The University of Edinburgh via the PCDS PhD studentship and LHCPhenoNet (Ø. A.), as well as the ERC starting grant "MathAm" (C. D.).

Note added in the proof.-Recently, we became aware of Ref. [66], which reports on the computation of the threeloop four-gluon amplitudes in $\mathcal{N}=4$ in super Yang-Mills. Upon specialising our result for $\Delta_{4}^{(3)}$ to the four-particle momentum-conserving limit they consider, Ref. [66] finds consistency with the infrared singularities of their amplitude, thus providing a check of both computations.

*On leave from the "Fonds National de la Recherche Scientifique" (FNRS), Belgium.

[1] A. H. Mueller, Phys. Rev. D 20, 2037 (1979).

[2] J. C. Collins, Phys. Rev. D 22, 1478 (1980). 
[3] A. Sen, Phys. Rev. D 24, 3281 (1981).

[4] A. Sen, Phys. Rev. D 28, 860 (1983).

[5] J. Gatheral, Phys. Lett. B 133, 90 (1983).

[6] J. Frenkel and J. Taylor, Nucl. Phys. B246, 231 (1984).

[7] G. P. Korchemsky and A. V. Radyushkin, Phys. Lett. B 171, 459 (1986); Nucl. Phys. B283, 342 (1987).

[8] G. P. Korchemsky and G. Marchesini, Phys. Lett. B 313, 433 (1993).

[9] G. P. Korchemsky, Phys. Lett. B 325, 459 (1994); I. A. Korchemskaya and G. P. Korchemsky, Phys. Lett. B 387, 346 (1996); Nucl. Phys. B437, 127 (1995).

[10] L. Magnea and G. F. Sterman, Phys. Rev. D 42, 4222 (1990).

[11] S. Catani and M. H. Seymour, Nucl. Phys. B485, 291 (1997); 510, 503(E) (1998).

[12] S. Catani, Phys. Lett. B 427, 161 (1998); G. F. Sterman and M. E. Tejeda-Yeomans, Phys. Lett. B 552, 48 (2003).

[13] L. J. Dixon, L. Magnea, and G. F. Sterman, J. High Energy Phys. 08 (2008) 022.

[14] N. Kidonakis, G. Oderda, and G. F. Sterman, Nucl. Phys. B531, 365 (1998).

[15] R. Bonciani, S. Catani, M. L. Mangano, and P. Nason, Phys. Lett. B 575, 268 (2003).

[16] Y. L. Dokshitzer and G. Marchesini, J. High Energy Phys. 01 (2006) 007.

[17] S. M. Aybat, L. J. Dixon, and G. F. Sterman, Phys. Rev. D 74, 074004 (2006).

[18] E. Gardi and L. Magnea, J. High Energy Phys. 03 (2009) 079.

[19] T. Becher and M. Neubert, Phys. Rev. Lett. 102, 162001 (2009); J. High Energy Phys. 06 (2009) 081.

[20] E. Gardi and L. Magnea, Nuovo Cimento Soc. Ital. Fis. C 32N5-6, 137 (2009).

[21] L. J. Dixon, Phys. Rev. D 79, 091501 (2009).

[22] L. J. Dixon, E. Gardi, and L. Magnea, J. High Energy Phys. 02 (2010) 081.

[23] V. Del Duca, C. Duhr, E. Gardi, L. Magnea, and C. D. White, Phys. Rev. D 85, 071104 (2012); J. High Energy Phys. 12 (2011) 021.

[24] S. Caron-Huot, J. High Energy Phys. 05 (2015) 093.

[25] V. Ahrens, M. Neubert, and L. Vernazza, J. High Energy Phys. 09 (2012) 138.

[26] S. G. Naculich, H. Nastase, and H. J. Schnitzer, J. High Energy Phys. 04 (2013) 114.

[27] O. Erdogan and G. Sterman, Phys. Rev. D 91, 065033 (2015).

[28] T. Gehrmann, E. Glover, T. Huber, N. Ikizlerli, and C. Studerus, J. High Energy Phys. 06 (2010) 094.

[29] N. Kidonakis, Phys. Rev. Lett. 102, 232003 (2009).

[30] A. Mitov, G. Sterman, and I. Sung, Phys. Rev. D 79, 094015 (2009).

[31] T. Becher and M. Neubert, Phys. Rev. D 79, 125004 (2009).

[32] M. Beneke, P. Falgari, and C. Schwinn, Nucl. Phys. B828, 69 (2010).

[33] M. Czakon, A. Mitov, and G. F. Sterman, Phys. Rev. D 80, 074017 (2009).

[34] A. Ferroglia, M. Neubert, B. D. Pecjak, and L. L. Yang, Phys. Rev. Lett. 103, 201601 (2009).

[35] A. Ferroglia, M. Neubert, B. D. Pecjak, and L. L. Yang, J. High Energy Phys. 11 (2009) 062.

[36] J.-y. Chiu, A. Fuhrer, R. Kelley, and A. V. Manohar, Phys. Rev. D 80, 094013 (2009).
[37] A. Mitov, G. F. Sterman, and I. Sung, Phys. Rev. D 82, 034020 (2010).

[38] E. Gardi, J. High Energy Phys. 04 (2014) 044; G. Falcioni, E. Gardi, M. Harley, L. Magnea, and C. D. White, J. High Energy Phys. 10 (2014) 10.

[39] J. M. Henn, A. V. Smirnov, and V. A. Smirnov, J. High Energy Phys. 07 (2013) 128.

[40] E. Gardi, E. Laenen, G. Stavenga, and C. D. White, J. High Energy Phys. 11 (2010) 155.

[41] A. Mitov, G. Sterman, and I. Sung, Phys. Rev. D 82, 096010 (2010).

[42] E. Gardi and C. D. White, J. High Energy Phys. 03 (2011) 079.

[43] E. Gardi, J. M. Smillie, and C. D. White, J. High Energy Phys. 09 (2011) 114.

[44] E. Gardi, J. M. Smillie, and C. D. White, J. High Energy Phys. 06 (2013) 088.

[45] G. F. Sterman, arXiv:hep-ph/9606312.

[46] S. Moch, J. A. M. Vermaseren, and A. Vogt, Nucl. Phys. B688, 101 (2004).

[47] A. Grozin, J. M. Henn, G. P. Korchemsky, and P. Marquard, Phys. Rev. Lett. 114, 062006 (2015).

[48] S. Moch, J. Vermaseren, and A. Vogt, Phys. Lett. B 625, 245 (2005).

[49] Ø. Almelid, C. Duhr, and E. Gardi (to be published).

[50] C. Anastasiou and A. Daleo, J. High Energy Phys. 10 (2006) 031; M. Czakon, Comput. Phys. Commun. 175, 559 (2006); A. V. Smirnov and V. A. Smirnov, Eur. Phys. J. C 62, 445 (2009); D. A. Kosower, BARNESROUTINES, http://projects .hepforge.org/mbtools/.

[51] C. Anastasiou, C. Duhr, F. Dulat, and B. Mistlberger, J. High Energy Phys. 07 (2013) 003.

[52] A. Goncharov, arXiv:0908.2238 [Adv. Math. (to be published)]; A. B. Goncharov, M. Spradlin, C. Vergu, and A. Volovich, Phys. Rev. Lett. 105, 151605 (2010); C. Duhr, H. Gangl, and J. R. Rhodes, J. High Energy Phys. 10 (2012) 075; C. Duhr, J. High Energy Phys. 08 (2012) 043; F. C. S. Brown, Ann. Sci. Ecole Norm. Sup. 42, 371 (2009); F. Brown, arXiv:1102.1310; Commun. Math. Phys. 287, 925 (2009); J. Ablinger, J. Blümlein, C. Raab, C. Schneider, and F. Wißbrock, Nucl. Phys. B885, 409 (2014); E. Panzer, Comput. Phys. Commun. 188, 148 (2015).

[53] F. C. S. Brown, C. R. Acad. Sci. Paris, Ser. I 338, 527 (2004).

[54] E. Remiddi and J. A. M. Vermaseren, Int. J. Mod. Phys. A 15, 725 (2000).

[55] L. J. Dixon, C. Duhr, and J. Pennington, J. High Energy Phys. 10 (2012) 074.

[56] See Supplemental Material at http://link.aps.org/ supplemental/10.1103/PhysRevLett.117.172002 for the expression for the function $F(z)$ of Eq. (14) in terms of HPLs.

[57] D. Gaiotto, J. Maldacena, A. Sever, and P. Vieira, J. High Energy Phys. 12 (2011) 011.

[58] F. Chavez and C. Duhr, J. High Energy Phys. 11 (2012) 114.

[59] V. A. Smirnov, Phys. Lett. B 567, 193 (2003).

[60] Z. Bern, L. J. Dixon, and V. A. Smirnov, Phys. Rev. D 72, 085001 (2005).

[61] Taking the Regge limit requires analytic continuation to the physical region of $2 \rightarrow 2$ scattering, to be discussed in detail in Ref. [49]. 
[62] S. Caron-Huot, E. Gardi, and L. Vernazza (to be published).

[63] Z. Bern, V. Del Duca, W. B. Kilgore, and C. R. Schmidt, Phys. Rev. D 60, 116001 (1999); D. A. Kosower, Nucl. Phys. B552, 319 (1999); I. Feige and M. D. Schwartz, Phys. Rev. D 90, 105020 (2014); S. Catani, D. de Florian, and G. Rodrigo, Phys. Lett. B 586, 323 (2004).
[64] We recall that strict collinear factorization is restricted to timelike kinematics with both collinear partons in the final state, but it is violated for spacelike splitting [65].

[65] S. Catani, D. de Florian, and G. Rodrigo, J. High Energy Phys. 07 (2012) 026.

[66] J. M. Henn and B. Mistlberger, this issue, Phys. Rev. Lett. 117, 171601 (2016). 